\title{
Canada's federal government should continue to proceed with caution on MAiD policy
}

\author{
Andreas Laupacis MD MSc
}

— Cite as: CMAJ 2020 February 24;192:E188-9. doi: 10.1503/cmaj.200213; early-released February 12, 2020

See related article at www.cmaj.ca/lookup/doi/10.1503/cmaj.200016

$\mathbf{T}$ he legalization of medical assistance in dying (MAiD) in June 2016 has been the most profound change in health care during my medical career. There is no more important and emotional time than someone's death, and how some Canadians could choose to die was fundamentally changed overnight.

The legalization of MAiD has challenged us to ensure, above all, that patients' wishes are fulfilled. At the same time, we have had to respect health care workers' personal views about the practice and ensure that MAiD is offered only to those who meet the criteria identified in the federal legislation. In Canada, MAiD can be provided only to someone who is clear of mind at the time of death, has an illness from which death is reasonably foreseeable, and who states that they have intolerable physical or psychologic suffering despite access to treatments for that suffering.

Canada's government is now considering whether to expand the criteria for access to MAiD. Therefore, Downar and colleagues' analysis of the first 2241 medically assisted deaths in Ontario is timely. ${ }^{1}$ The authors found that those who received MAiD were more likely to have cancer, be married and live in a wealthy neighbourhood than all people who died in Ontario. Three-quarters of people who received MAiD were cared for by palliative care practitioners before their death. The 10-day reflection period before the procedure was shortened for $58 \%$ of those whose estimated prognosis was less than 1 month. Family or providers reported concerns about difficulties in accessing MAiD for $7 \%$ of deaths.

These results are largely reassuring. It is important that we collect and publicly report detailed information about all who receive MAiD in Canada. Other provinces should release similar reports.

Downar and colleagues' findings should allay concerns that people living in Ontario are choosing MAiD because they have little social support or have poor access to health care. This study found the opposite, which suggests that more attention needs to be paid to ensure that those who are socially or economically vulnerable and eligible for MAiD are aware that MAiD is an option.
It is encouraging that $58 \%$ of people with an estimated prognosis of less than 1 month had providers who were willing and able to shorten the statutory 10 -day reflection period so that they could have the type of death they wanted. Perhaps the 10-day reflection period should be abandoned for those close to death. Information about how often people who were approved for MAiD changed their minds during those 10 days would be helpful in addressing that question.

Although concerns about difficulties accessing MAiD were infrequent, the study provided no information about people who may have encountered so much difficulty that they could not access the procedure at all. This should be an area of future inquiry. Information about people admitted to faith-based institutions, who should have the same timely access to MAiD as everyone else, was also lacking.

The approach the government of Canada took when it first legalized MAiD can be characterized as "proceed with caution, measure what we are doing, and assess." That was the right approach. At least in Ontario, the first assessment is reassuring.

However, we appear to be entering a new era regarding MAiD in Canada. The Superior Court of Quebec recently ruled that it was unconstitutional for MAiD to be denied to 2 people who are living with severe disabilities because their death was not reasonably foreseeable. ${ }^{2}$ Stimulated by this decision, the government of Canada has indicated its commitment to expanding eligibility for MAiD beyond those who are nearing the end of life. ${ }^{3}$ The government is also considering whether eligibility for MAiD should be expanded to 3 other groups of Canadians: mature minors, people for whom mental illness is their only reason for requesting MAiD, and advance requests for those who anticipate that they may lose the capacity to request it in the future (e.g., owing to severe dementia).

More than a year ago, the Council of Canadian Academies issued thorough and thoughtful reports about these 3 conditions. ${ }^{4}$ Although there is limited international experience to learn from, it is clear that important ethical concerns related to the particular vulnerability of patients in these 3 groups must be addressed.

Allowing mature 17-year-old Canadians suffering intractably from advanced cancer to have access to MAiD should require relatively minor changes to current safeguards. However, providing 
MAiD to socially isolated Canadians who are living with advanced chronic diseases or severe disabilities from which death is not imminently foreseeable is a different matter. Access to highquality health care can sometimes be difficult for them, and we must ensure that such patients receive high-quality care before MAiD is contemplated.

If access to MAiD is expanded, new safeguards, specifically tailored to each new indication for MAiD, should be put in place. Then, we must once more proceed with caution, measure carefully and reassess.

\section{References}

1. Downar J, Fowler RA, Falko R, et al. Early experience with medical assistance in dying in Ontario, Canada: a cohort study. CMAJ 2020;192:E173-81.

2. Jessome J. Canada: Truchon v. Procureur général du Canada: Superior Court of Quebec finds limiting access to medical assistance in dying ("MAiD") to end of life unconstitutional. mondaq; 2019. Available: www.mondaq.com/canada/Food
-Drugs-Healthcare-Life-Sciences/855424/Truchon-v-Procureur-Gnral-Du-Canada -Superior-Court-Of-Quebec-Finds-Limiting-Access-To-Medical-Assistance-In-Dying -Maid-To-End-Of-Life-Unconstitutional (accessed 2020 Feb. 6).

3. Government of Canada consults Canadians on medical assistance in dying [news release]. Ottawa: Department of Justice Canada; 2020. Available: www.canada.ca/ en/department-justice/news/2020/01/government-of-canada-consults-canadians -on-medical-assistance-in-dying.html (accessed 2020 Feb. 6).

4. The Expert Panel on Medical Assistance in Dying. State of knowledge on medical assistance in dying for mature minors, advance requests, and where a mental disorder is the sole underlying medical condition: summary of reports. Ottawa: Council of Canadian Academies; 2018. Available: https://cca-reports.ca/wp -content/uploads/2018/12/MAID-Summary-of-Reports.pdf (accessed 2020 Feb. 6).

Competing interests: See www.cmaj.ca/site/misc/cmaj_staff.xhtml

Affiliation: Editor-in-chief, CMAJ

Correspondence to: CMAJ editor, cmaj@cmajgroup.ca 